\title{
Computational modelling approaches to vaccinology
}

\author{
Francesco Pappalardo ${ }^{1}$, Darren Flower ${ }^{2}$, Giulia Russo ${ }^{1}$, Marzio Pennisi ${ }^{3}$, \\ Santo Motta ${ }^{3}$ \\ ${ }^{1}$ Dipartimento di Scienze del Farmaco - \\ University of Catania, Catania, Italy \\ ${ }^{2}$ School of Life and Health Sciences \\ Aston University, Aston Triangle, Birmingham, UK \\ ${ }^{3}$ Dipartimento di Matematica e Informatica - \\ University of Catania, Catania, Italy
}

\begin{abstract}
Excepting the Peripheral and Central Nervous Systems, the Immune System is the most complex of somatic systems in higher animals. This complexity manifests itself at many levels from the molecular to that of the whole organism. Much insight into this confounding complexity can be gained through computational simulation. Such simulations range in application from epitope prediction through to the modeling of vaccination strategies. In this review, we evaluate selectively various key applications relevant to computational vaccinology: these include technique that operates at different scale i.e., from molecular to organisms and even to population level.
\end{abstract}

Keywords: vaccine research; modelling; computational vaccinology; immune system; epitopes; simulations.

\section{Introduction}

Despite its overwhelming and often confounding complexity, the immune system is ultimately a collection of parts working together to effect defence against pathogens 
and many other homeostatic functions. The problem, of course, when one tries to understand the immune system, is the remarkable level of emergent behaviour we observe - at many levels - from the formation of supramolecular complexes at the Immune synapse; through the action of specific immune cells, such as dendritic cells and T-cells; to organs; and, finally, whole organisms. Beyond even the whole animal, effects such as herd immunity and infectivity patterns manifest themselves only in large, interacting pseudo-social networks. As we see, or allude to, elsewhere, much of this can be modelled, and modelled with some success. Yet despite the daunting emergent, higher-level behaviour we see, much can still be learned from attempting to understand and model the underlying molecular components that comprise the immune system.

Nowadays biological systems are analysed and managed by means of new emerging technologies that are revolutionizing biotechnology and information technology, producing a huge amounts of data. This data needs to be integrated and is quickening the process of knowledge discovery, enabling the study of biological systems at various levels i.e., from molecules to organisms and even to the population level.

The human activity entailing the representation, the manipulation and the communication of real-world daily life objects is known as modelling. Mathematical and computational models are gradually used to assist deduce biomedical data produced by high-throughput genomics and proteomics endeavours. The application of advanced computer models allowing the simulation of complex biological processes produces hypotheses and proposes experiments. Computational models are set to exploit the wealth of data stored on biomedical databases through text mining and knowledge discovery methods.

The first immunoinformatics tools for vaccine design were developed in the 1980s by DeLisi and Berzofsky and others [56]. Chief among vaccine design informatics tools are epitope-mapping algorithms. A new era of vaccine research began in 1995, when the complete genome of Haemophilus influenzae (a pathogenic bacterium) was published [58]. In parallel with advances in molecular biology and sequencing technology, bioinformatics analysis of microbial genome data has allowed in silico selection of vaccine targets. Further advances in the field of immunoinformatics have led to the development of hundreds of new vaccine design algorithms. This novel approach for developing vaccines has been named reverse vaccinology [59] or immunome-derived vaccine design [60]. Pharmaceutical companies are starting to use 
models to optimize/predict therapeutic effects at the organism level, suggesting that computational biology can effectively play a key role in this field [57].

Along with these techniques, the simulation of the immune system in a detailed way to reproduce and predict the effects of artificial immunity elicited by vaccines represents a challenge that several people are attempting with success. The immune system represents one of the most complex biological system. It is, in fact, an adaptive learning system which operates at multiple levels (molecules, cells, organs, organisms, and groups of organisms). Immunological research, both basic and applied, needs to deal with this complexity [4].

In this paper, we analyse and discuss several computational modelling techniques applied to vaccinology science.

\section{Epitopes}

Arguably, the simplest unambiguous component of the immune is the so-called epitope. The epitope at its most generally defined is very much the immunological quantum that lies central to immune responses and vaccination. It is the ability of the immune system to identify, respond to, and remember epitopes that powers natural immunity, and thus vaccination. Peptide epitopes are mediated primarily by their interaction with Major Histocompatibility Complexes (T-cell Epitopes, or TCEs) and antibodies (B-cell epitopes, or BCE).

Currently, commonly-used prediction of B cell epitopes often remains primitive, or depends on an elusive knowledge of protein structure, and both structure- [9] and datadriven [10] prediction of antibody-mediated epitopes have again been shown to be poor. Explaining such sub-optimality may point to a fundamental misinterpretation of extant epitope data. PEPSCAN is perhaps the most abundant data available currently but may not be what it seems. Experimentally derived epitopes are identified by assayed against pre-existing antibodies with affinity for whole antigens. If, for example, "epitopes" are mapped back to their original antigen structure, we find them randomly located through the structure rather than equating to obvious surface patches, as might be expected if they simply reproduced discontinuous epitopes identified by crystallography. In situ antigenic regions are often not exposed and thus accessible to binding by antibodies binding but rather completely buried. If we compare the conformation of antibody-bound peptides with those from the intact antigen, they are usually quite different. However, B-cell epitopes in isolated antigen and in whole antigen-antibody complexes are much more similar. Is it possible then, that the isolated 
peptide adopts a conformation which mimics the surface features of a discontinuous epitope or that the preformed antibody recognize denatured antigen in vivo.

Currently, prediction of $\mathrm{T}$ cell epitopes remains largely confined to predictions of varying accuracy of peptide binding to Major Histocompatibility Complex. Nonetheless, and compared to B-cell prediction, methods for predicting $\mathrm{T}$ cell epitopes show significant algorithmic sophistication. Prediction of the binding of peptides to class I MHCs, at least for well-studied alleles, such as HLA-A*0201, is now at useable accuracy [11]. However, comparative studies have shown recently that the prediction of class II MHC binding prediction T-cell epitopes is typically poor [12][13][14][15], and likewise for structure-driven prediction of class I and class II T-cell epitopes [16].

All epitope prediction methods remain severely constrained by the data used to construct them; this is particularly true of T-cell prediction. It has recently been shown that that T-cell epitopes, which were previously thought to be short peptides of 8-10 amino acids, can be up to 16 amino acids or perhaps even more. The existence of such longmer epitopes has significantly enlarged the repertoire of peptides open to inspection by T-cells [17]. Many of the cutting edge approaches to epitope discovery are trying to address these issues by inducing models of large numbers of alleles across many peptide lengths by making assumptions about how separable are the sub-sites in the peptide binding groove and how thee can be combined combinatorially to generate pseudo-binding profiles [18][19][20]. However, as is well-known, no datadriven method can go beyond the data used to train it; all methods are likewise much superior in their ability to interpolate than their ability to extrapolate.

Evidence exists that the responsiveness of the immune system to pathogenic proteins is only poorly correlated with the possession of $\mathrm{T}$ cell epitopes, and that many potential epitopes have been deleted in proteins regularly accessible to immune surveillance, perhaps as an evolutionary counter measure in the war between host and pathogen [21]. Such a deficit, and the significantly sub-optimal prediction of both B-cell and T-cell epitopes described above have suggested that methods which rely solely on the possession of epitopes are unlikely to be effective at identifying antigens or immunogens. This conjecture is confirmed by what information there is, which indicates that there is little simple correspondence between antigens selected on this basis and experimentally verified antigenic or protective proteins. In turn this has led to the development of other approaches to predicting whole antigens within pathogen genomes, proteins likely to be antigenic and protective; of which there are three key approaches: subcellular location prediction, sequence similarity, and empirical 
statistical approaches, typified by VaxiJen [22][23] and expert systems such as nerve [24].

\section{PAMPS and Adjuvants}

Other epitopes exist, notable the so-called Pathogen-Associated Molecular Pattern (or PAMP), highly conserved and typically complex molecular moieties recognised by pattern recognition receptors (or PRRs) of the innate immune system [25]. Many PAMPs, and molecules mimicking the recognition of PAMPs, form the basis of adjuvants. Adjuvants potentiate immune responses, reducing the dosing requirements needed to induce protective immunity, particularly for weakly immunogenic subunit vaccines. Few adjuvants are licensed for human use: principally alum, and squalenebased oil-in-water adjuvants. Yet there are many types of potential adjuvant, including proteins, oligonucleotides, drug-like small-molecules, and liposome-based delivery systems.

So-called Small Molecule Adjuvants (SMAs) are the most underexplored of existing adjuvants, despite the observation that many small molecules exhibit overt adjuvanticity. SMAs include both complex biologically-derived natural products and fully-synthetic drug-like molecules [26]. Notable natural Product SMAs include QS21; muramyl dipeptide; various formulations of mannide monooleate, MurNAc-L-Ala- $\gamma-D-$ Glu-mDAP (M-TriDAP), and Monophosphoryl-Lipid A (or MPL).

Fully-synthetic drug-like small molecules are also adjuvants [26]: for example, Bestatin (Ubenimex or UBX), Levamisole; Bupivacaine; and 2-(4-Methoxyphenyl)-Nmethylethanamine also known as compound 48/80. Yet easily the best explored of SMAs are the so-called Imidazoquinolines, the best known are Imiquimod, Resiquimod, and Gardiquimod, these target Toll-like receptors: TLR7 and/or TLR8, inducing IFN, TNF and IL-12 secretion.

SMAs can also be discovered systematically using virtual screening approaches [26]: the best example is our discovery of adjuvants functioning as antagonists of the CCR4 Chemokine receptor. Inhibiting CCR4 receptors may give rise to adjuvantism as the receptor is expressed by regulatory T-cells (or Tregs) that normally suppress immune responses [27]. Inhibiting CCR4 function is anticipated to exacerbate vaccine responses. By combining experimental validation with virtual screening, we have identified several potential adjuvants, acting through the apparent inhibition of Treg proliferation [28][29]. Three-dimensional or structure-based virtual screening (SB-VS), which utilises automated protein docking (APD), is an effective means of identifying 
ligands with great celerity [30][31][32]. VS can identify real, high-affinity ligands with unmatched cost-effectiveness and efficiency. APD-based SB-VS can dock innumerable ligand molecules into a defined binding site [33]. SB-VS is exceptionally logistically efficient, saving time, labour, and resource. Months of robotically-mediated experimentation are replaced by weeks of computational analysis, complemented by a handful of reliable, hand-crafted assays. At most a few hundred molecules need be tested [33]. This handful is put through a hierarchical cascade of highly specific and informative assays in vitro, with actives then tested for their whole system adjuvant properties in vivo. The molecules we found [28][29] behave appropriately in a variety of in vitro assays, and increase the levels of various correlates of protection in vaccinated mice, and even show some enhancement in related challenge models observations supported by independent analysis [34]; and also shows activity against potential cancer antigens [35].

\section{Higher order systems}

Much of what we have discussed above has focussed on the analysis of experimental structures and sequences. Although 3D modelling of epitope MHC and epitope-antibody interactions have occasionally been illuminating, other uses of explicit 3D modelling of immune receptors has been more edifying and successful.

A key example is provided by the trimeric MHC-peptide-TCR (pMHC-TCR) complex, a supramolecular complex at the heart of the cellular immune response [36]. Small molecule drugs can block allele specific peptide presentation to T-cells, which is both a potential mechanism to exploit therapeutically [37][38] and a pathological mechanism leading to so-called Adverse Drug Actions [39][40]. For many Adverse Drug Reactions (ADRs), particularly cutaneous ADRs, there is a strong association between the reaction to certain drugs (including abacavir, allopurinol, carbamazepine, and other antiepileptic drugs) and particular HLA alleles, allowing for the prognostic prediction of ADRs. There is likewise a potential relationship between the haplotype of donor and recipient and the outcomes of stem-cell and solid organ transplants.

Establishing relationships for these rare events is complicated by the $3000+$ different MHC alleles known to exist in the global human population, which in its turn leads to the extraordinary potential for distinct peptide specificities within the global patient population. Each MHC allele has a unique sequence, and thus unique 3-dimensional structure and functional properties, including their binding specificity for peptides and TCRs; and it is possible to compare MHC structure as a way to classify them in terms 
of such important functional interactions. We have recently used poisson-boltzmann electrostatic potential as a meaningful arbiter of through-space molecular interactions combined with sophisticated data-mining methods to address this. By rigorous state-ofthe-art analysis of projected properties, we identified clusters corresponding to the three class I human $\mathrm{MHC}$ loci, and sub groups therein. It is notable that this recovers the HLA-A; HLA-B, and HLA-C alleles without any prior knowledge of such a division. This gives confidence to any assertion we might make regarding the other division of the allele population into structurally and functionally similar sub-groups. Supertype analysis has potential applications in the classification of MHC specificity for peptide and TCR interaction, with implications therefore for epitope prediction, solid organ and bone marrow transplantation, mate-choice, and MHC-mediated adverse drug reactions.

Molecular dynamic simulations have long been applied to attempting to unravel the many mysteries of the immune system [41]. Powered by the availability of supercomputing MD simulations can now tackle very large systems [42]. Yet even the largest immunological simulation is small compared to such biomolecular simulations. Assisted by rapid advances in experimental imaging and quantitative proteomics, simulations begin to approach the mesoscale [43]; and we can look to simulate in a reasonably realistic way biologically meaningful cellular events. For example, we have simulated at atomic resolution a detailed molecular model of part of the immune synapse, comprising CD4, peptide-MHC, TCR, and membrane regions [42]. MD will ultimately break free from the many restrictions imposed by the limited data we currently have, allowing us the luxury of de novo prediction of equilibrium binding and kinetic constants. Beyond that, we can envisage conducting simulations that pose biological questions that can only be answered by experiment, which in turn will drive the design of experiments.

\section{Agent based models in computational vaccinology}

Computational models are important for the understanding of biological systems. Such models can be applied to enhance or predict therapeutic effects at the organism level. The pharmaceutical companies suggest that computational biology can play an excellent role in this field [44]. In silico models can afford answers to the general behaviour of the immune system, the analysis of cellular and molecular interactions, the effects of treatments, and the course of diseases.

The use of agent based modelling (ABM) is suitable both to perform in silico 
experiments which lead to formulate and/or validate biological hypotheses and to give useful hints for the design of optimal treatment schedules. Moreover, ABM approach can be used at cellular level to describe complex systems in a flexible way, including the handling of entity heterogeneity and physical space. ABMs aim at recreate and predict the cellular interactions simulating the behaviour and the interactions of autonomous entities (cells and molecules). The dynamic agents can be described as a function of time, a position, and an internal state that includes most important properties of the agent, such as age.

One of the successful applications of $A B M$ in computational vaccinology is represented by SimTriplex [1], a specialized cellular automaton able to model mammary carcinoma, Triplex vaccine and the immune system competition. Triplex is an immunopreventive HER-2/neu breast cancer vaccine [2], which combines the specific target antigen, p185(HER-2/neu) with two non-antigen specific adjuvants: IL-12 and allogeneic major histocompatibility complex (MHC) class I molecules. Four vaccine administration schedules (early, late, very late and chronic) have been tested on HER$2 /$ neu transgenic mice, and the chronic schedule showed that it is the only one that provides complete, long- term protection from mammary carcinoma.

SimTriplex mimics the behaviour of immune cells at the cellular level in both vaccinated and in naive mice. The simulator incorporates a variety of cellular and molecular entities, including tumour and vaccine cells. Modifications of state (e.g., cell activation, cytotoxicity, cell death, etc.) are ruled by a set of policies based on tumour immunology. The model coupled with optimization techniques (based on combinatorial optimization algorithms as genetic algorithms and simulated annealing [7][8]) allowed to search for an optimal vaccination schedule to obtain the same efficacy of the chronic protocol with a definitively reduced vaccine administrations. SimTriplex predictions have been verified in a in-vivo experiment. Outcomes show that in-silico predicted schedule does significantly reduce the tumours multiplicity on the ten mice mammary glands even if the vaccination efficacy for the first appearing of tumour was still overestimated. Further adjustment of the model is required to include evidence of immune aging which appeared from in vivo follow up results [3][4].

The Triplex vaccine proved to be effective also as a therapeutic vaccine, showing its ability to be used against induced lung metastases [5]. A major goal of biologists is to better understand the biological behaviour to improve the efficacy of the therapeutic treatment and to try to predict, for example, the outcomes of longer experiments in order to move faster towards clinical phase I trials. In a recent work [6], the authors 
present MetastaSim, an ABM to be used as an in silico virtual lab with the target to help answering these questions. MetastaSim has the ability to simulate the cancer growth kinetics and multiple different metastatic nodules, each one with its own growth rate, in an accurate way. To reproduce the growth in time of nodules, the Gompertz growth law is used in its differential form. Simulations results showed that it is possible to obtain in silico a reduction of approximately $45 \%$ in the number of vaccinations. Most of the protocols presented there share a similar vaccination strategy that is composed by a boost of three vaccine injections, a period of rest, and then a series of vaccine recalls that are somewhat equally spaced. The model suggests that any optimal protocol for preventing lung metastases formation should be therefore composed by an initial massive vaccine dosage followed by few vaccine recalls. Even if this is a wellknown vaccination strategy in immunology, since it is commonly used for many infectious diseases such as tetanus and hepatitisB, it can be still considered a relevant result in the field of cancer-vaccines immunotherapy.

Vaccination strategies can be also used as therapeutics solutions. Immunological therapy of progressive tumours, in particular, requires both the activation and expansion of tumour specific cytotoxic $T$ lymphocytes, and an efficient migration of these effector cells in the tumour tissue.

In order to investigate in silico the melanoma progression and the effects of a therapeutic vaccination strategy against such tumour, an ABM named SimB16 has been realized and presented in [45].

SimB16 has been initially validated using in vivo results, and then used to predict the critical role of CD137 expression on tumour vessel endothelium. Thus it allowed to analyse the effects of anti-CD137 mAb derived by adoptive transfer of activated OT-1 T cells in B16-OVA mice.

Predictions show that early infiltration of $T$ cells seems to be dependent on CD137 expression on tumor vasculature, an this represents an important factor that must be taken into account in order to understand in vivo results and to design future administration strategies.

In [46] Kim and Lee present a hybrid ABM-delay differential equation (DDE) model to reproduce the general behaviour of preventative cancer vaccines (particularly cytotoxic T lymphocytes (CTLs)) in order to obtain Anti-Tumour Immunity. The model does not tackle a specific in vivo problem, but tries to theoretically understand the feasibility of CTL based vaccines. According to the model, an anti-cancer memory CTL pool of approximately $3 \%$ can successfully eradicate a tumour population under a wide 
range of biological hypotheses and scenarios, implying that a vaccination approach is feasible. Moreover, the model reveals some conditions that may entitle rapid tumour destruction, oscillation, and polynomial rather than exponential decline in the tumour population.

Another ABM-based approach to simulate vaccination in cancer immunotherapy is given by the Vacclmm Server [47]. This online model represents the first tool that tries to enable the simulation at a cellular scale of peptide vaccination using real amino acid sequences to reproduce molecular binding sites. Simulations can be executed directly online, and results allow to analyse the parameter space of the involved entities and to reveal the complex and patient-specific nature of peptidic vaccination in cancer immunotherapy. It must be said that Vacclmm uses classical molecular prediction tools in order to determine the affinity between receptors, so its results highly depend on the accuracy of such molecular tools.

\section{Mathematical models in computational vaccinology}

Mathematical models have been used since many years to represent various aspects of immune system and related pathologies, however their application to describe the effects of vaccines has been quite limited.

These models are mainly based on differential equations (ordinary, partial, delay, and/or stochastic) and are applied to describe the dynamics of immune system entities, cells, pathogens and treatments from a population point of view, rather than follow such entities individually, as seen in ABMs.

They are built on a strong and solid mathematic theory, and for simple models (i.e. with a limited number of simple equations) it is possible to find an analytical solution. However, for more complex models, the use of computational techniques that are focused in finding an approximate solution is mandatory.

The trade-off between tractability (and solvability) versus biological coherence usually tends on the former, thus such models are usually less accurate in describing the immunological background than ABMs. However their application to some specific problems allows to extract some fundamental properties, to study the parameter's space, and to provide sensitivity analysis. For example, the same problems tackled by SimTriplex and SimB16 ABMs have been explored from a qualitative point of view by ordinary and delay differential equation (ODE and DDE) based models [48][49][50].

In [51] Davis et al. present a mathematical model based on DDE to model shigella 
immune dynamics. The model does not care into account a specific vaccine, but it is used to determine which immune system responses must be stimulated by any candidate vaccine. According to the model, antibody-based vaccines targeting only surface antigens cannot elicit sufficient immunity for protection. However, boosting antilipopolisaccaride (LPS) B memory cells can help and give protection against shigella. Furthermore an extension of the model reveals that targeting both LPS and epithelial entry proteins could represent a favourable approach in designing new vaccines.

Differential equation based models have been mainly applied to cancer vaccinology. Papalardo et al. [52] developed an ordinary differential equation (ODE) based model to evaluate the number and the frequency of vaccine boosts needed to obtain a long-lasting and protecting memory T-cell response. The model includes both activated cytotoxic T lymphocytes and memory $T$ cells, and is used to investigate the induction of immunological memory in wild-type mice injected with a dendritic cellbased vaccine, both in the presence and in the absence of memory $T$ cells. A good agreement between ex vivo and in silico experiments underlines how the model is able to reproduce the expansion and persistence of antigen-specific memory $\mathrm{T}$ cells. Moreover statistical sensitivity analysis allowed the identification of a time window in which boosts may be detrimental.

Parra-Guillen et al. [53] use an approach based on an incremental series of steps to develop a mathematical model which describes the tumour response in mice after vaccination. The authors investigate its applicability to study cytokine-based strategies that can modulate the immune system response. In order to successfully describe the different outcomes obtained after vaccine administration different models have been integrated and used: (1) A Model of tumour growth in mice without treatment using a linear model; (2) A Model of the vaccine effects assuming that the vaccine triggers a delayed immune response that leads to cancer cells death (using two compartments); (3) A Model to reproduce a resistance effect that decreases the vaccine efficiency based on the size of tumour; (4) a mixture model to represent the relapse of the tumour, an event that has been observed in a small percentage of animals.

A mathematical model based on ODE has been developed by Wilson and Levy in order to gain insights about the combined effects of anti-TGF- $\beta$ treatments and vaccines against tumours. The mathematical model takes into account the dynamics of the tumour growth, the concentration of TGF- $\beta$, the action of activated cytotoxic effector and regulatory T cells. No treatment, anti-TGF- $\beta$ treatment, vaccine treatment, 
and combined anti-TGF- $\beta$ vaccine treatments scenarios are investigated through numerical simulations and stability analysis. The model well reproduces the observed experimental results, and could be in principle used to help the design of new treatments that include TGF- $\beta$.

Another example of the application of mathematical models to cancer vaccinology is represented by the work by Joshi et al. [55]. The authors present mathematical model based on ODE of therapeutic vaccination against cancer, and focus on the role of antigen presentation and co-stimulatory signalling pathways. The effects of different vaccination protocols on the well-documented phenomena of cancer dormancy and recurrence have been studied by means of numerical simulations. Results suggest a possible explanation of why adoptive immunotherapies can indeed sometimes promote tumour growth. Moreover simulations suggest that an elevated number of professional APCs well correlate with prolonged time periods of cancer dormancy.

\section{Conclusions}

Complexity is the hall mark of many somatic systems; not least the Immune system. Computational approaches are finally beginning to shine an illuminating light on how the Immune System functions at many levels, peeling away the obfuscating layers that have hitherto obscured our understanding. The functioning and mis-functioning of the Immune system lies at the heart of defence against infection and cancer and the induction of autoimmune disorders respectively. The ability to interact with the immune system through vaccination has created the most efficacious and efficient intervention in medical history, saving uncountable millions of lives across hundreds of years. The proven ability of computation to design vaccines and adjuvants, and to optimize vaccination protocols is beginning to open up a new era of computational vaccinology that will in time bring in its wake untold benefits to the burgeoning global population both through human vaccination but also in combating climate change and protecting livestock and aquaculture.

At the molecular level, the potent combination of data-driven machine learning methods and Molecular Dynamics-based atomistic simulation have allowed the development of various approaches that address a variety of key applications. Some have proved successful, including $T$ cell-mediated epitope prediction for well-studied alleles, while others have yet to deliver on their true potential, such as the prediction of 
antibody-mediated B-cell epitope prediction, which currently is often more misleading than helpful. One of the most promising approaches is the use of large scale dynamics simulations of cellular systems that can explore the behaviour of complex systems that currently lie beyond the power of experimental biophysics to properly evaluate. While we are at least several decades away from simulating a whole eukaryotic cell, it is now possible to simulate supramolecular systems of hitherto inconceivable size and complexity using multi-scale approaches that combine both atomistic and various levels of course-grained simulations.

\section{References}

[1] Pappalardo F, Lollini PL, Castiglione F, Motta S. Modeling and simulation of cancer immunoprevention vaccine. Bioinformatics 2005; 21(12):2891-97.

[2] Nanni P, Nicoletti G, De Giovanni C, et al. Combined allogeneic tumor cell vaccination and systematic interleukin 12 prevents mammary carcinogenesis in HER-2/neu transgenic mice. Journal of Experimental Medicine 2001;194(9): 1195-1205.

[3] Palladini A, Nicoletti G, Pappalardo F, et al. In silico modeling and in vivo efficacy of cancer-preventive vaccinations. Cancer Research 2010;70(20): 7755-63.

[4] Pappalardo F, Pennisi M, Castiglione F, Motta S. Vaccine protocols optimization: in silico experiences. Biotechnology Advances 2010;28(1):82-93.

[5] Nanni P, Nicoletti G, Palladini A, et al. Antimetastatic activity of a preventive cancer vaccine. Cancer Research 2007;67(22)11037-44.

[6] Pennisi M, Pappalardo F, Palladini A, et al. Modeling the competition between lung metastases and the immune system using agents. BMC Bioinformatics 2010;11(7-S13).

[7] Pappalardo F, Mastriani E, Lollini PL, Motta S. Genetic algorithm against cancer. Lecture Notes in Computer Science 2006;3849:223-28.

[8] Pennisi M, Catanuto R, Pappalardo F, Motta S. Optimal vaccination schedules using simulated annealing. Bioinformatics 2008;24(15):1740-42.

[9] Ponomarenko JV, Bourne PE. Antibody-protein interactions: benchmark datasets and prediction tools evaluation. Bmc Struct Biol. 2007;7:64.

[10] Blythe MJ, Flower DR. Benchmarking B cell epitope prediction: underperformance of existing methods. Protein Sci. 2005;14:246-248.

[11] Lafuente EM, Reche PA. Prediction of MHC-peptide binding: a systematic and 
comprehensive overview. Curr Pharm Des. 2009;15:3209-3220.

[12] Gowthaman U, Agrewala JN. In silico tools for predicting peptides binding to HLA-class II molecules: more confusion than conclusion. J Proteome Res. 2008;7:154-163.

[13] El-Manzalawy Y, Dobbs D, Honavar V. On evaluating MHC-II binding peptide prediction methods. Plos One. 2008;3:e3268.

[14] Lin HH, Zhang GL, Tongchusak S, Reinherz EL, Brusic V. Evaluation of MHC-II peptide binding prediction servers: applications for vaccine research. Bmc Bioinformatics. 2008;9(Suppl 12):S22.

[15] Zhang H, Wang P, Papangelopoulos N, Xu Y, Sette A, Bourne PE, Lund O, Ponomarenko J, Nielsen M, Peters B. Limitations of Ab initio predictions of peptide binding to MHC class II molecules. Plos One. 2010;5:e9272.

[16] Knapp B, Omasits U, Frantal S, Schreiner W. A critical cross-validation of high throughput structural binding prediction methods for pMHC. J Comput Aided Mol Des. 2009;23:301-307.

[17] Macdonald IK, Harkiolaki M, Hunt L, Connelley T, Carroll AV, MacHugh ND, Graham SP, Jones EY, Morrison WI, Flower DR, Ellis SA. MHC class I bound to an immunodominant Theileria parva epitope demonstrates unconventional presentation to T cell receptors. PLoS Pathog. 2010 Oct 14;6(10):e1001149

[18] Jørgensen KW, Rasmussen M, Buus S, Nielsen M. NetMHCstab - predicting stability of peptide-MHC-I complexes; impacts for cytotoxic T lymphocyte epitope discovery. Immunology. 2014 Jan;141(1):18-26.

[19] Karosiene E, Rasmussen M, Blicher T, Lund O, Buus S, Nielsen $M$. NetMHCllpan-3.0, a common pan-specific MHC class II prediction method including all three human MHC class II isotypes, HLA-DR, HLA-DP and HLADQ. Immunogenetics. 2013 Oct;65(10):711-24.

[20] Karosiene E, Lundegaard C, Lund O, Nielsen M. NetMHCcons: a consensus methodfor the major histocompatibility complex class I predictions. Immunogenetics. 2012 Mar;64(3):177-86.

[21] Halling-Brown M, Shaban R, Frampton D, Sansom CE, Davies M, Flower D, Duffield M, Titball RW, Brusic V, Moss DS. Proteins accessible to immune surveillance show significant T-cell epitope depletion: Implications for vaccine design. Mol Immunol. 2009 Aug;46(13):2699-705.

[22] Doytchinova IA, Flower DR. Identifying candidate subunit vaccines using an alignment-independent method based on principal amino acid properties. 
Vaccine. 2007 Jan 15;25(5):856-66.

[23] Doytchinova IA, Flower DR. VaxiJen: a server for prediction of protective antigens, tumour antigens and subunit vaccines. BMC Bioinformatics. 2007 8:4.

[24] Ivona S, Bernante F, Filippini F. NERVE: new enhanced reverse vaccinology environment. BMC Biotechnol. 2006;6:35.

[25] Bianchi, M.E., DAMPs, PAMPs and alarmins: all we need to know about danger. J Leukoc Biol, 2007. 81(1): p. 1-5.

[26] Flower, D.R., Systematic identification of small molecule adjuvants. Expert Opin Drug Discov, 2012. 7(9): p. 807-17.

[27] Kim, C.H., Migration and function of Th17 cells. Inflamm Allergy Drug Targets, 2009. 8(3): p. 221-8.

[28] Bayry, J., et al., From 'perfect mix' to 'potion magique'-- regulatory T cells and anti-inflammatory cytokines as adjuvant targets. Nat Rev Microbiol, 2008. 6(1): p. C1; author reply C2.

[29] Bayry, J., et al., In silico identified CCR4 antagonists target regulatory T cells and exert adjuvant activity in vaccination. Proc Natl Acad Sci U S A, 2008. 105(29): p. 10221-6.

[30] Mclnnes C: Virtual screening strategies in drug discovery. Current opinion in chemical biology 2007, 11(5):494-502.

[31] Ripphausen P, Nisius B, Peltason L, Bajorath J: Quo vadis, virtual screening? A comprehensive survey of prospective applications. J Med Chem 2010, 53(24):8461-8467.

[32] Bajorath J: Computational studies, virtual screening, and theoretical molecular models. J Med Chem 2010, 53(1):1-2.

[33] Hattotuwagama CK, Davies MN, Flower DR: Receptor-ligand binding sites and virtual screening. Current medicinal chemistry 2006, 13(11):1283-1304.

[34] Davies, M.N., et al., Toward the discovery of vaccine adjuvants: coupling in silico screening and in vitro analysis of antagonist binding to human and mouse CCR4 receptors. PLoS One, 2009. 4(11): p. e8084.

[35] Pere, H., et al., A CCR4 antagonist combined with vaccines induces antigenspecific CD8+ T cells and tumor immunity against self antigens. Blood, 2011. 118(18): p. 4853-62.

[36] Michels, A.W., Targeting the trimolecular complex. Clin Immunol, 2013.

[37] Michels, A.W., et al., Structure-based selection of small molecules to alter allele-specific MHC class II antigen presentation. J Immunol, 2011. 187(11): p. 
5921-30.

[38] Michels, A.W., et al., Identification of Small Molecules That Enhance AntiInsulin Peptide T Cell Receptor Signaling and IL-10 Secretion. Diabetes, 2010. 59: p. A89-A89.

[39] Aihara, M., Pharmacogenetics of cutaneous adverse drug reactions. J Dermatol, 2011. 38(3): p. 246-54.

[40] Hausmann, O., B. Schnyder, and W.J. Pichler, Etiology and pathogenesis of adverse drug reactions. Chem Immunol Allergy, 2012. 97: p. 32-46.

[41] Flower DR, Phadwal K, Macdonald IK, Coveney PV, Davies MN, Wan S. T-cell epitope prediction and immune complex simulation using molecular dynamics: state of the art and persisting challenges. Immunome Res. 2010 Nov 3;6 Suppl 2:S4.

[42] Wan S, Flower DR, Coveney PV. Toward an atomistic understanding of the immune synapse: large-scale molecular dynamics simulation of a membraneembedded TCR-pMHC-CD4 complex. Mol Immunol. 2008 Mar;45(5):1221-30.

[43] Sanbonmatsu KY, Tung CS. High performance computing in biology: Multimillion atom simulations of nanoscale systems. Journal of Structural Biology. 2007;157(3):470-480.

[44] Price M. Computational Biologists: The Next Pharma Scientists? Science Carrier Magazine. 2012;doi:10.1126/science.caredit.a1200041.

[45] Pappalardo F, Forero IM, Pennisi M, et al. SimB16: Modeling Induced Immune System Response against B16-Melanoma. PLoS ONE 2012;6(10):e26523. doi:10.1371/journal.pone.0026523.

[46] Kim PS, Lee PP. Modeling Protective Anti-Tumor Immunity via Preventative Cancer Vaccines Using a Hybrid Agent-based and Delay Differential Equation Approach, PLoS Comput Biol 2012;8(10):e1002742. doi:10.1371/journal.pcbi.1002742

[47] Von Eichborn J, Woelke A-L, Castiglione F, Preissner R. Vacclmm: Simulating peptide vaccination in cancer therapy, BMC Bioinformatics 2013; 14:127

[48] Bianca C, Pennisi M. The triplex vaccine effects in mammary carcinoma: A nonlinear model in tune with SimTriplex, Nonlinear Analysis: Real World Applications 2012, 13(4):1913-1940

[49] Bianca C, Chiacchio F, Pappalardo F, Pennisi M. Mathematical modeling of the immune system recognition to mammary carcinoma antigen, BMC Bioinformatics 2012, 13 Supp.17, S21 
[50] Pennisi M. A Mathematical Model of Immune System-Melanoma Competition, Computational and Mathematical Methods in Medicine, 2012, Article ID 850754, 13 pages doi:10.1155/2012/850754.

[51] Davis CL, Wahid R, Toapanta FR, Simon JK, Sztein MB, Levy D. Applying Mathematical Tools to Accelerate Vaccine Development: Modeling Shigella Immune Dynamics, PLoS ONE 2013; 8(4): e59465. doi:10.1371/journal.pone.0059465

[52] Pappalardo F, Pennisi M, Ricupito A, Topputo F, Bellone M. Induction of T cell memory by a dendritic cell vaccine: a computational model, Bioinformatics 2014, 30(13):1884-1891, doi:10.1093/bioinformatics/btu059.

[53] Parra-Guillen ZP, Berraondo P, Grenier E, Ribba B, Troconiz IF. Mathematical Model Approach to Describe Tumour Response in Mice After Vaccine Administration and its Applicability to Immune-Stimulatory Cytokine-Based Strategies, The AAPS Journal 2013; 15(3): 797-807

[54] Wilson S, Levy D. A Mathematical Model of the Enhancement of Tumor Vaccine Efficacy by Immunotherapy, Bull Math Biol 2012; 74:1485-1500, DOI $10.1007 / \mathrm{s} 11538-012-9722-4$

[55] Joshi B, Wanga X, Banerjeea S, Tianb H, Matzavinos A, Chaplain MAJ. On immunotherapies and cancer vaccination protocols: A mathematical modelling approach, Journal of Theoretical Biology 2009, 259:820-82

[56] DeLisi C, Berzofsky JA. T-cell antigenic site stend to be amphipathic structures, Proceedings of the National Academy of Sciences of the United States of America 1985; 82(20):7048-7052

[57] Kumar N, Hendriks BS, Janes KA, de Graaf D, Lauffenburger DA. Applying computational modeling to drug discovery and development, Drug Discov Today 2006;11(17/18):806-11.

[58] Fleischmann RD, Adams MD, White $O$ et al. Whose genome random sequencing and assembly of Haemophilus influenzae Rd, Science 1995; 269(5223):496-521.

[59] Rappuoli R. Reverse vaccinology, Current Opinion in Microbiology 2000; 3(5): 445-450

[60] De Groot AS, McMurry J, Moise L, Martin B. Epitope-based Immunome-derived vaccines: a strategy for improved design and safety, Applications of Immunomics, A. Falus, Ed., Springer Immunomics Series, Springer, New York, NY, USA, 2009. 


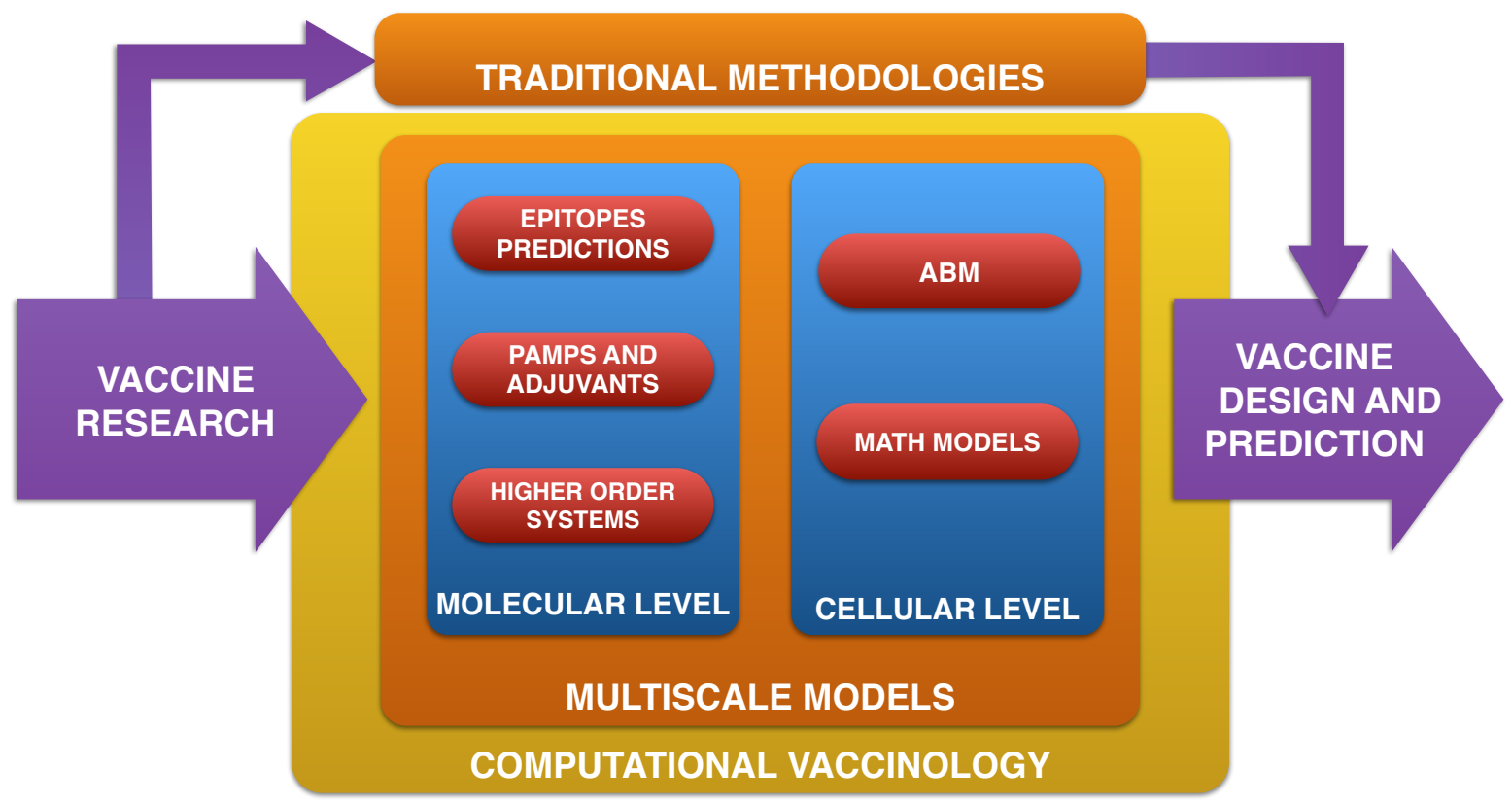

Figure 1 - Graphical abstract. The functioning and mis-functioning of the Immune system lies at the heart of defence against infection and cancer and the induction of autoimmune disorders respectively. Nowadays, traditional methodologies in vaccine research are combined with computational vaccinology i.e., computational strategies to design vaccines and adjuvants, and to optimize vaccination protocols. These methodologies act at different levels and one the most ambitious goal is to have them integrated together to reach a multiscale view and approach. 